\title{
A Green Synthesis of 3, 4-Dihydropyrimidin-2(1H)-ones via One-Pot Multi-Component Reaction by Using Cuttlebone as a Natural Catalyst under Solvent-Free Conditions
}

\author{
Nooshin Firoozeh, Soodabeh Rezazadeh* and Cobra Izanloo \\ Department of Chemistry, Faculty of Sciences, Bojnourd Branch, Islamic Azad University, Bojnourd 9417697796, Iran. \\ soodabehrezazadeh@yahoo.com
}

Received February 14 ${ }^{\text {th }}, 2017$; Accepted May $12^{\text {th }}, 2017$.

\begin{abstract}
In this paper, an efficient, green and eco-friendly method for synthesis of 3,4-dihydropyrimidin-2(1H)-ones via one-pot multi-component reaction of aldehydes, urea and ethyl acetoacetate is shown at the presence of cuttlebone as an effective, reusable and natural heterogeneous catalyst. The advantages of this method are short reaction times, high yields, simple work-up, and reusable natural catalysts. The structure of cuttlebone was characterized by FT-IR spectrum, scanning electron microscopy (SEM) and energy dispersive spectrometer (SEM-EDS).
\end{abstract}

Key words: Cuttlebone; 3, 4-dihydropyrimidin-2(1H)-ones; aldehydes; one-pot reaction; green.

\section{Introduction}

3, 4-Dihydropyrimidin-2(1H)-ones are an important class of heterocyclic compounds in the area of natural and synthetic organic chemistry. They possess a variety curative and pharmacological applications involving antiviral, antitumor, antibacterial and anti-inflammatory activities [1-3]. Moreover, these compounds have appeared as potential calcium channel blockers [4], antihypertensive [5], $\alpha 1$ a-adrenergic antagonists [6] and neuropeptide antagonists [7].

Multi-component reactions (MCRs) are one-pot reactions in which three or more starting materials reacted to form a product. MCRs display high atom economy and high selectivity [8-10]. The advantages of these reactions involve: synthetic productivity, simplicity of procedure, decreasing number of isolation and purification steps, save on energy, time and costs and also reduction of waste [11-14]. The synthesis of 3, 4-dihydropyrimidin-2(1H)-ones (DHPMs) which was initially reported by Pietro Biginelli is one of the excellent MCRs that includes a three-component one-pot reaction of an aldehyde, ethyl acetoacetate and urea in the presence of strong acid [15]. The disadvantages of classical Biginelli reaction include hard reaction conditions, long reaction times and low yields [16-18]. In recent years, new methods for preparation of DHPMs have been developed to improve and modify this reaction using microwave irradiation [19], ultrasound irradiation [20], ionic liquids
Resumen: En este documento, se muestra un método eficiente, agradable para el medio ambiente y ecológico para la síntesis de 3,4-dihidropirimidin-2 $(1 H)$-onas, mediante la reacción de multicomponentes de aldehídos, urea y acetato de etilo en presencia de "cuttlebone" como un catalizador heterogéneo natural, eficaz y reutilizable. Unas de las ventajas de este método son: tiempos cortos de reacción, alta eficiencia, procedimiento fácil y el empleo de un catalizador natural y reutilizable. La estructura del "cuttlebone" fue caracterizada por FT-IR, microscopía electrónica de barrido (SEM) y espectrometría de dispersión de energía (SEM-EDS).

Palabras clave: Cuttlebone; 3, 4-dihidropirimidin-2(1H)-onas; aldehídos; reacción en un solo recipiente; química verde.

[21], Lewis and Bronsted acid such as lanthanide triflate [22], $\mathrm{H}_{3} \mathrm{BO}_{3}$ [23], $\mathrm{VCl}_{3}$ [24], $\mathrm{Sr}(\mathrm{OTf})_{2}$ [25], $\mathrm{Bi}_{2}\left(\mathrm{SO}_{4}\right)_{3}$ [26], Indium(III) halides [27], $\mathrm{KAl}\left(\mathrm{SO}_{4}\right)_{2} \cdot 12 \mathrm{H}_{2} \mathrm{O}$ supported on silica [28], silicasulfuric acid [23,29], $\mathrm{Mn}(\mathrm{OAc})_{3} \cdot 2 \mathrm{H}_{2} \mathrm{O}$ [30], $\mathrm{Y}\left(\mathrm{NO}_{3}\right)_{3} \cdot 6 \mathrm{H}_{2} \mathrm{O}$ [31], zeolite [32], $\mathrm{TaBr}_{5}$ [33], $\mathrm{Ce}\left(\mathrm{NO}_{3}\right)_{3} \cdot 6 \mathrm{H}_{2} \mathrm{O}$ [34], glutamic acid [35], $\mathrm{H}_{7}\left[\mathrm{PMo}_{8} \mathrm{~V}_{4} \mathrm{O}_{40}\right.$ ] [36], lemon juice [37] and so on. However, some of the methods are presented to have one or more drawbacks such the use of expensive reagents, harmful to the environment, strong acidic conditions, long reaction times and low yields. Due to the importance of DHPMs, the introduction of mild and green methods with higher yields are needed.

Green chemistry is the model of chemical products and processes that reduce or eliminate the use or generation of hazardous substance. Nowadays the development of eco-friendly and economical processes is a challenge for chemists. Replacement of toxic, polluting and non-recyclable catalysts with eco-friendly reusable solid, heterogeneous catalysts are an area of current interest [38].

According to the above points, in this research, we want to report a convenient and green method for the synthesis of 3,4-dihydropyrimidin-2(1H)-ones via the one-pot multi-component reaction of aldehydes with ethyl acetoacetate and urea at the presence of cuttlebone as an eco-friendly, recyclable and heterogeneous natural catalyst under solvent-free conditions (Scheme 1). 
<smiles></smiles>

R: $\mathrm{C}_{6} \mathrm{H}_{5}, 4-\mathrm{OHC}_{6} \mathrm{H}_{4}, 4-\mathrm{MeC}_{6} \mathrm{H}_{4}, 3-\mathrm{OMeC}_{6} \mathrm{H}_{4}, 3-\mathrm{ClC}_{6} \mathrm{H}_{4}, 3-\mathrm{BrC}_{6} \mathrm{H}_{4}, 4-\mathrm{FC}_{6} \mathrm{H}_{4}$ '

$1 \mathrm{a}-\mathrm{I}$ 4- $\mathrm{NO}_{2} \mathrm{C}_{6} \mathrm{H}_{4}$ ' furyl, trans-cinnamyl, propyl, methyl

Scheme 1. The synthesis of 3, 4-dihydropyrimidin-2(1H)-ones using cuttlebone as a green catalyst.

\section{Results and Discussion}

In this paper, we describe a simple and efficient protocol for the synthesis of 3,4-dihydropyrimidin-2(1H)-ones via one-pot multi-component condensation of aldehydes, ethyl acetoacetate and urea using cuttlebone as a novel, reusable and eco-friendly heterogeneous natural catalyst.

\section{Characterization of cuttlebone}

Cuttlebone is the calcic internal shell of a cuttlefish. The cuttlebone is a class of ultra-lightweight cellular natural material that possess unique chemical, mechanical and structural properties such as high rigidity, high porosity and high penetrance. The dorsal shield and the lamellar matrix are two main components of cuttlebone [39]. The dorsal shield has a hard and stiff structure that provides a rigid stratum for protection of the structure and the development of the lamellar matrix of cuttlebone $[40,41]$. The lamellar matrix of cuttlebone has the highest degree of porosity (up to $90 \%$ ), but also can tolerate high hydrostatic pressure. The lamellar matrix consists primarily of aragonite (a crystallized form of calcium carbonate, $\mathrm{CaCO}_{3}$ ), covered in a layer of organic material [40-42] composed of $\beta$-chitin [43]. Cuttlebone that is also known as a cuttlefish bone (Sepia esculenta) [44-47] is commonly found on saltwater beaches like the Persian Gulf in Iran.

The FT-IR spectrum of powdered cuttlebone is shown in Fig. 1. The absorption bands of planar $\mathrm{CO}_{3}{ }^{-2}$ ion (aragonite) are observed at 1082, 857, 1478 and $700 \mathrm{~cm}^{-1}$. Also, the board bands at about $3500-3300 \mathrm{~cm}^{-1}$ are attributed to the $\mathrm{OH}$ and $\mathrm{NH}$ stretching vibrations of $\beta$-chitin segment of cuttlebone, respectively. In fact, the hydrogen bonding network of $\beta$-chitin of cuttlebone cause to broadening absorption bands of $\mathrm{OH}$ and $\mathrm{NH}$ groups. The absorption bands of symmetric stretching of $\mathrm{CH}$, $\mathrm{CH}_{3}$ and asymmetric stretching vibrations of $\mathrm{CH}_{2}$, are appeared from 2929 to $2855 \mathrm{~cm}^{-1}$. In addition, the stretching vibration of $\mathrm{C}=\mathrm{O}$ of amide at $1654 \mathrm{~cm}^{-1}$ and the stretching vibrations of asymmetric bridge oxygen and $\mathrm{C}-\mathrm{O}$ at 1160 to $1030 \mathrm{~cm}^{-1}$, are observed [48-50].

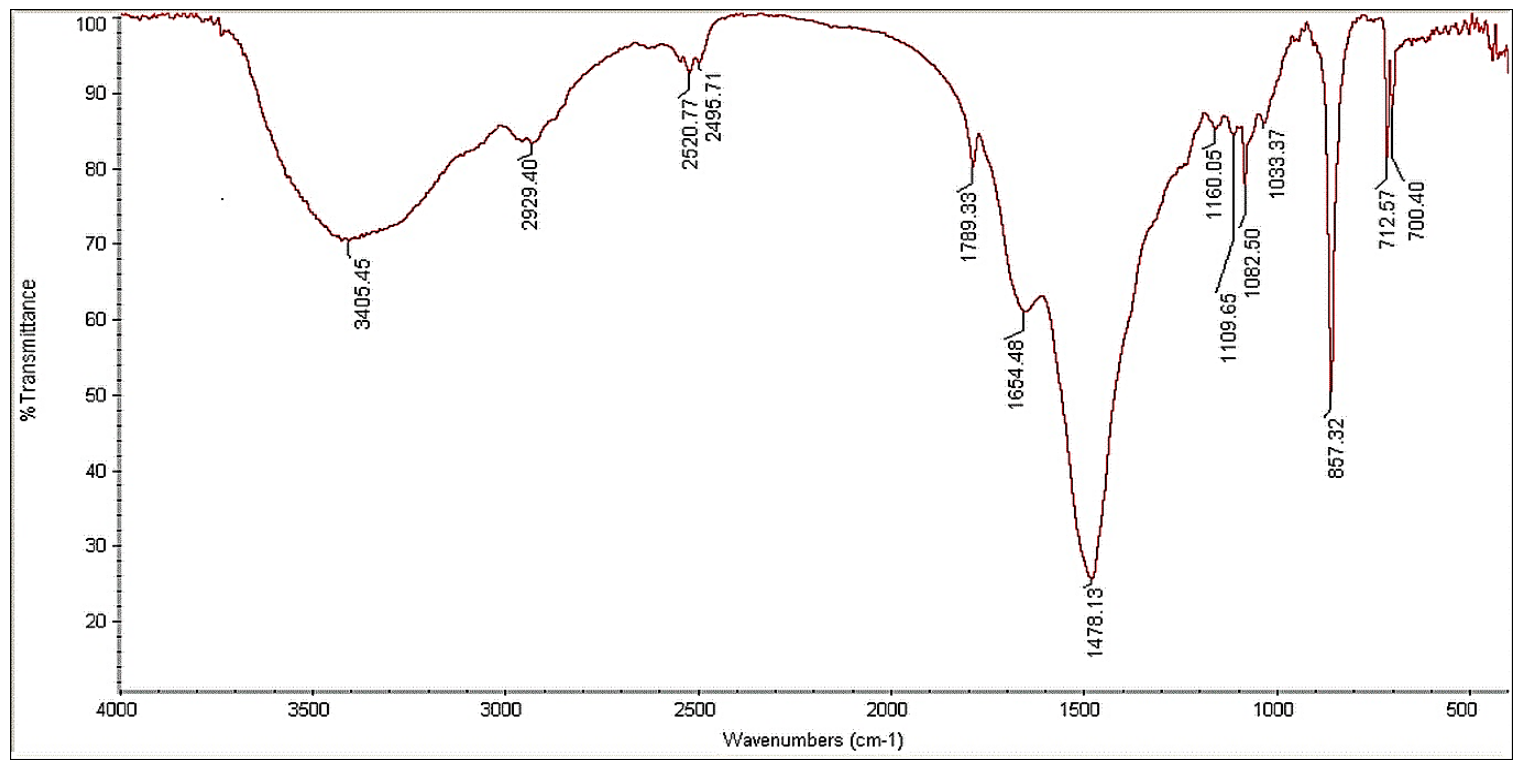

Fig. 1. The FT-IR spectrum of powdered cuttlebone. 

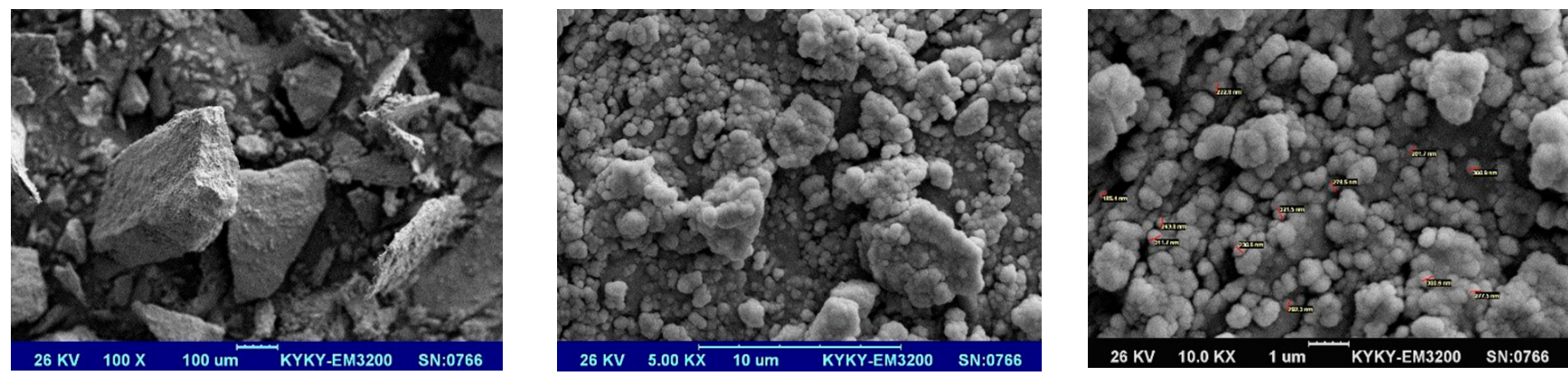

Fig. 2. The SEM images of powdered cuttlebone.

Scanning electronic microscopy (SEM) of powdered cuttlebone shows an amorphous morphology with a 185-321 nm particle size (Fig. 2).

Fig. 3 shows the energy dispersive spectrum (EDS) of powdered cuttlebone. According to this spectrum, the cuttlebone structure consists of $\mathrm{Ca}, \mathrm{C}$ and $\mathrm{O}$ elements.

In order to find the best reaction conditions, the one-pot three-component reaction of benzaldehyde, ethyl acetoacetate and urea was chosen as a model reaction (Scheme 2).
At first the model reaction was studied at the presence of 0.1 gr of some natural catalysts such as cuttlebone, calcined eggshell (CES) and natural zeolite under solvent-free conditions at $100{ }^{\circ} \mathrm{C}$ (Table 1 , entries 1-3). As Table 1 (entry 1) shows the use of cuttlebone is more efficient for the model reaction. Then, the different amounts of cuttlebone was tested on the model reaction (Table 1, entries 1 and 4-5). According to the results, increasing the amount of catalyst has no effect on yield of product, while a decrease of the amount of the catalyst cause

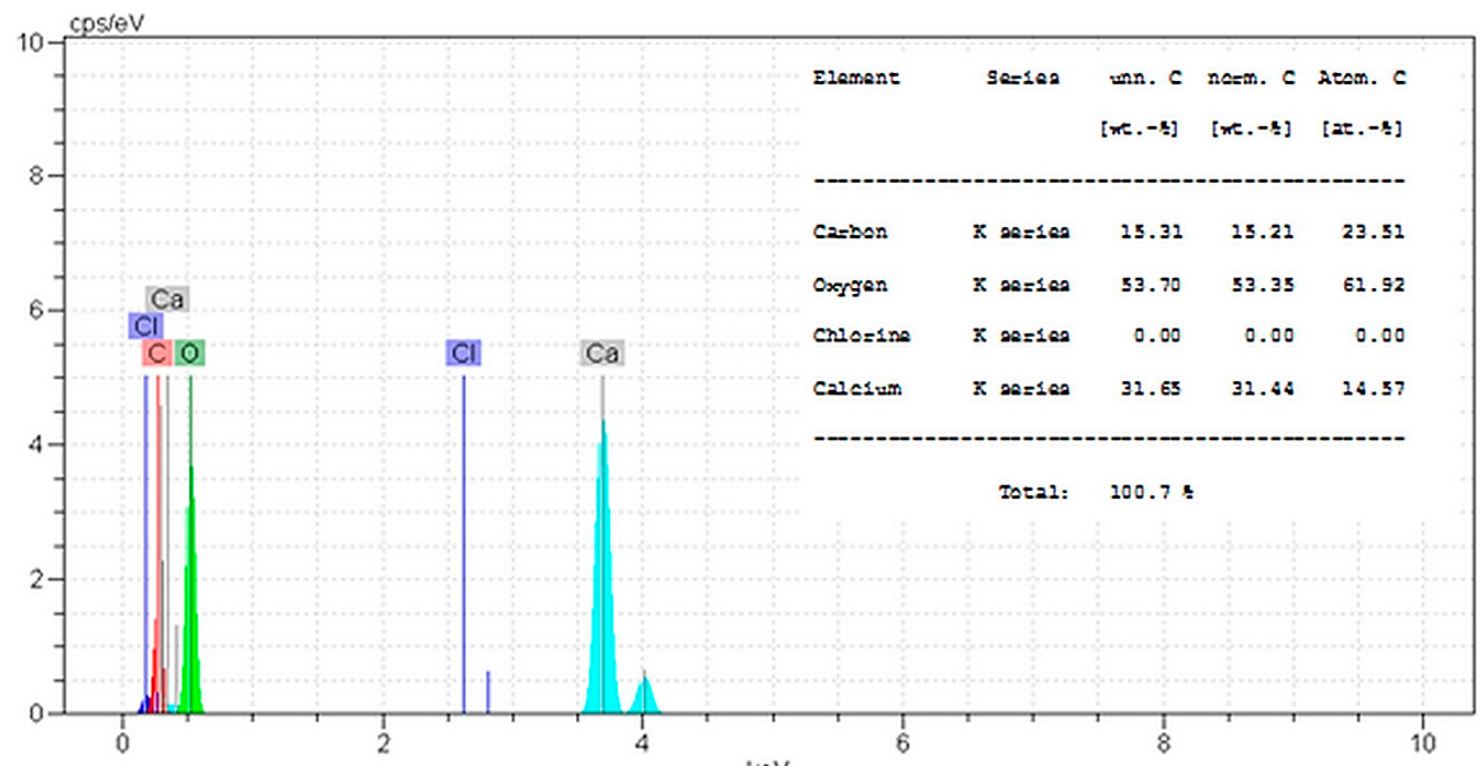

Fig. 3. The EDS spectrum of powdered cuttlebone.<smiles>O=Cc1ccccc1</smiles>

A<smiles>CCOC(=O)CC(C)=O</smiles>

B<smiles>NC(N)=O</smiles>

C<smiles>CCOC(=O)C1=C(C)NC(=O)NC1c1ccccc1</smiles>

$1 \mathrm{a}$

Scheme 2. The one-pot three-component reaction of benzaldehyde, ethyl acetoacetate and urea under different conditions. 
Table 1. The effect of varying conditions on the model reaction.

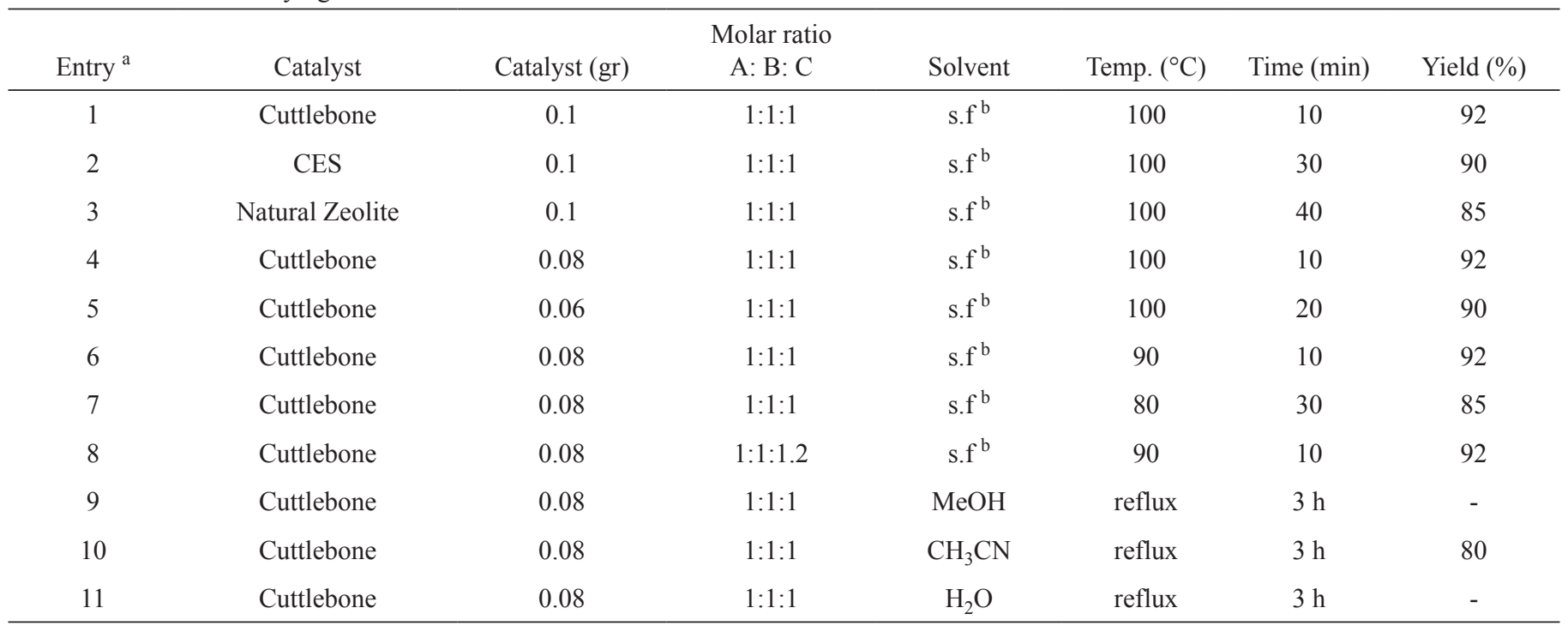

${ }^{\mathrm{a}}$ Isolated yield; ${ }^{\mathrm{b}}$ Solvent-free.

of decreasing desired product (1a). After that, the model reaction was carried out under different temperature at the presence of $0.08 \mathrm{gr}$ of catalyst (Table 1 , entries $4,6-7$ ). The results showed that the reducing the temperature to $90{ }^{\circ} \mathrm{C}$ had no effect on yield and reaction time. Applying 1: 1: 1.2 molar ratio of benzaldehyde: ethyl acetoacetate: urea at the presence of 0.08 gr of catalyst under solvent-free conditions had no effect on yield and reaction time (Table 1, entries 6 and 8). Finally, to investigate the effect of solvent, the model reaction was performed in presence and absence of solvent (Table 1, entries 6 and 9-11). The best results were obtained with 0.08 gr of cuttlebone under solvent-free conditions at $90{ }^{\circ} \mathrm{C}$ (Table 1 , entry 6).

To indicate the scope and the generality of the method, eth$\mathrm{yl}$ acetoacetate was reacted with aromatic, heteroaromatic and aliphatic aldehydes with a wide range of functional groups and urea under an optimized reaction conditions to giving 3, 4-dihydropyrimidin-2(1H)-ones with good to high yields $(75-95 \%)$. The results are presented in Table 2.

Aromatic aldehydes with unsubstituted/substituted electron-donating and electron-withdrawing groups such as -OH, $-\mathrm{CH}_{3},-\mathrm{OCH}_{3},-\mathrm{Cl},-\mathrm{Br},-\mathrm{F},-\mathrm{NO}_{2}$ gave excellent yields of corresponding products (Table 3 , entries 1-8). Furthermore, $\alpha, \beta$-unsaturated aldehydes and heteroaromatic aldehydes also afforded good yields (Table 3, entries 9 and 10). Interestingly, aliphatic aldehydes also gave desired products in good yields. (Table 3, entries 11 and 12).

To check the recyclability of catalyst, after completion of the reaction of benzaldehyde, ethyl acetoacetate with urea, the catalyst was separated from the reaction mixture, washed with ethanol and distilled water several times to remove the organic products, and dried. The heterogeneous catalyst was used for 3 successive times in the new experiments obtaining good yields (Fig. 4).

\section{Conclusion}

We have developed a novel and green protocol for the synthesis of 3, 4-dihydropyrimidin-2(1H)-ones via one-pot multi-component reaction. Generality, efficiency, simple work-up procedure and purification, recyclability of the catalyst, short reaction times, high yields, low cost and finally conformity with the green chemistry principles are the advantages of this protocol.

\section{Experimental}

The chemicals were purchased from Merck. Melting points of products were determined with an Electrothermal Type 9100 melting point apparatus. The FT-IR spectra were recorded on an Avatar 370 FT-IR Thermo Nicole spectrometer. The NMR spectra were determined on a Bruker Avance $300 \mathrm{MHz}$ instrument in DMSO- $d_{6}$. Elemental analyses were performed using an Elementar, Vario EL III and Thermofinnigan Flash EA 1112 Series instrument. All the reactions were monitored by thin layer chromatography (TLC); the spots were visualized with UV light. Scanning electron microscopy (SEM) and energy dispersive spectrometry (SEM-EDS) were performed on a KYKYEM3200 operated at a $30 \mathrm{KV}$ accelerating. All of the products were known compound and characterized by the FT-IR spectroscopy. The structure of products was further confirmed by ${ }^{1} \mathrm{H}$ NMR and ${ }^{13} \mathrm{C}$ NMR spectroscopy. Cuttlebone, that is also known as cuttlefish bone (Sepia esculenta) [44-47] is commonly found on saltwater beaches like the Persian Gulf in Iran.

\section{Pre-preparation of cuttlebone}

In order to the use of cuttlebone in reaction, at first the catalyst was powdered, then washed with distilled water to remove 
Table 2. Synthesis of 3, 4-dihydropyrimidin-2(1H)-ones (1a-11) catalyzed by cuttlebone under solvent-free conditions.

\begin{tabular}{|c|c|c|c|c|}
\hline Entry & Aldehyde & Product & Time $(\min )$ & Yield $^{\mathrm{a}}(\%)$ \\
\hline 1 & $\mathrm{O}$ & $1 \mathrm{a}$ & 10 & 92 \\
\hline 2 & $\mathrm{CHO}$ & 1b & 30 & 90 \\
\hline 3 & $\mathrm{CHO}$ & 1c & 60 & 95 \\
\hline 5 & & $1 e$ & 15 & 94 \\
\hline 6 & $\mathrm{CHO}$ & $1 \mathrm{f}$ & 15 & 95 \\
\hline
\end{tabular}


Table 2. Synthesis of 3, 4-dihydropyrimidin-2(1H)-ones (1a-11) catalyzed by cuttlebone under solvent-free conditions.

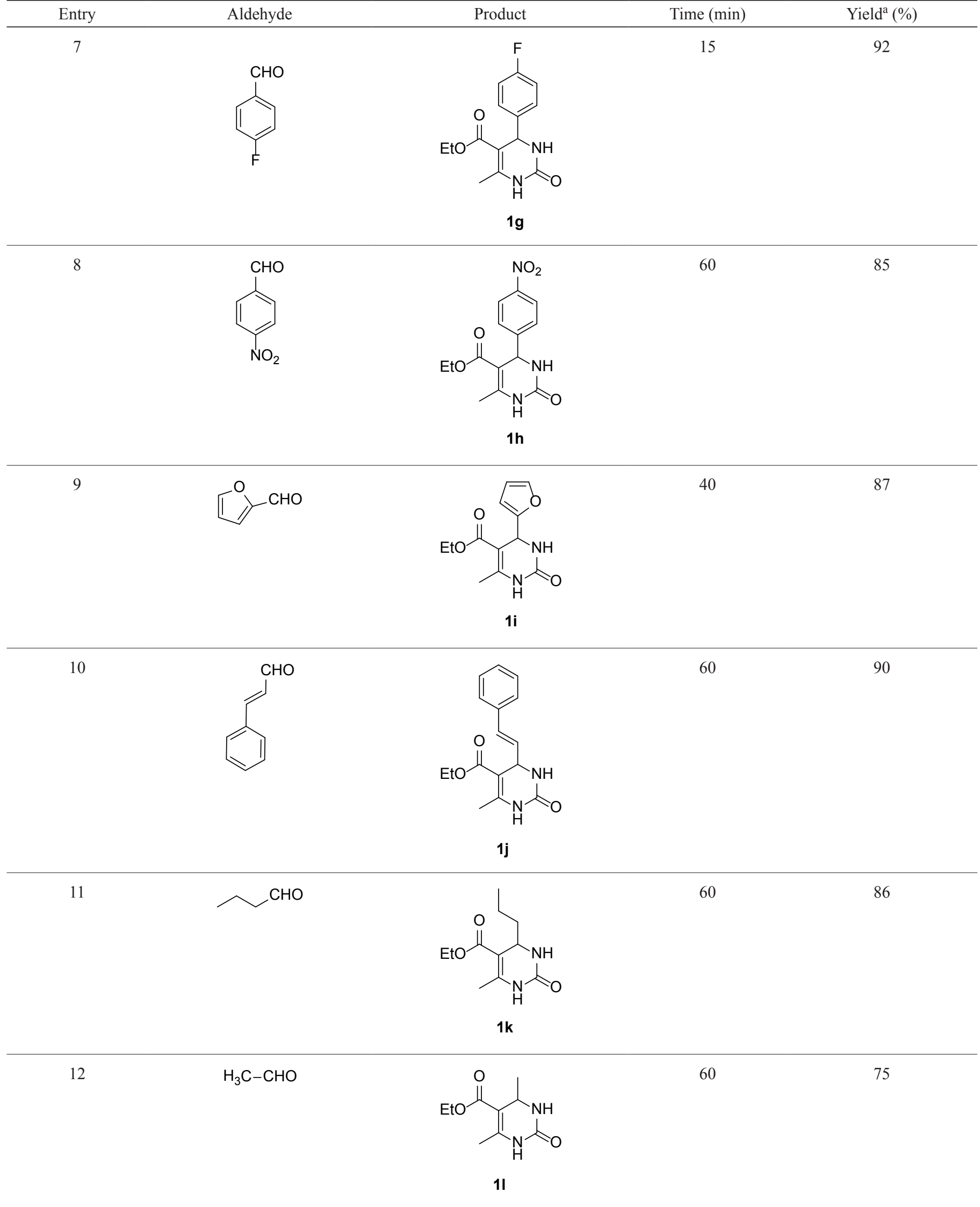




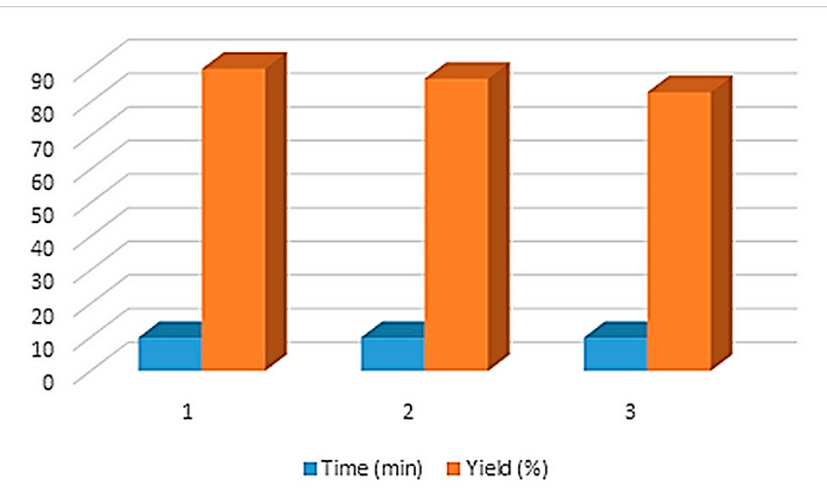

Fig. 4. Synthesis of 5-ethoxycarbonyl-4-phenyl-6-methyl-3, 4-dihydropyrimidin-2(1H)-one (1a) in the presence of reused catalyst.

pollution on the surface of cuttlebone and finally dried at 100 ${ }^{\circ} \mathrm{C}$ for $2 \mathrm{~h}$ [48].

Typical procedure for the preparation of 5-(ethoxycarbonyl)-6-methyl-4-phenyl-3, 4-dihydropyrimidin-2(1H)-one (1a).

A mixture of benzaldehyde ( $1 \mathrm{mmo1}, 0.106 \mathrm{gr}$ ), ethyl acetoacetate $(1 \mathrm{mmol}, 0.147 \mathrm{gr})$, urea $(1 \mathrm{mmol}, 0.060 \mathrm{gr})$ and cuttlebone $(0.08 \mathrm{gr})$ was heated at $90{ }^{\circ} \mathrm{C}$ in an oil bath for $10 \mathrm{~min}$. After completion of the reaction as monitored by TLC, the reaction mixture was cooled to room temperature, diluted with ethanol $(10 \mathrm{~mL})$ and filtered to recover the catalyst. The organic layer was separated and concentrated under reduced pressure. The crude product was recrystallized from ethanol to afford 0.241 gr $1 \mathbf{a}(92 \%)$.

5-(Ethoxycarbonyl)-6-methyl-4-phenyl-3,4-dihydropyrimidin-2(1H)-one (1a). White solid; mp 206-208 ${ }^{\circ} \mathrm{C}$ (Lit. 206-208 ${ }^{\circ} \mathrm{C}$ ) [51]; FT-IR (KBr) $v_{\max } 3244,3116,2978,2937,1725,1702$, 1648, 1596, 1465, 1421, 1313, 1221, 1090, 1026, 783, 757, $699,661,516 \mathrm{~cm}^{-1} ;{ }^{1} \mathrm{H}$ NMR $\left(300 \mathrm{MHz}, \mathrm{DMSO}-d_{6}\right) \delta 9.22$ $(1 \mathrm{H}, \mathrm{s}, \mathrm{NH}), 7.77(1 \mathrm{H}, \mathrm{s}, \mathrm{NH}), 7.25-7.36\left(5 \mathrm{H}, \mathrm{m}, \mathrm{C}_{6} \mathrm{H}_{5}\right), 5.16$ $(1 \mathrm{H}, \mathrm{d}, J=3.0 \mathrm{~Hz}, \mathrm{CH}), 4.00\left(2 \mathrm{H}, \mathrm{q}, J=6.0 \mathrm{~Hz}, \mathrm{OCH}_{2} \mathrm{CH}_{3}\right)$, $2.27\left(3 \mathrm{H}, \mathrm{s}, \mathrm{CH}_{3}\right), 1.11\left(3 \mathrm{H}, \mathrm{t}, J=6.0 \mathrm{~Hz}, \mathrm{OCH}_{2} \mathrm{CH}_{3}\right) ;{ }^{13} \mathrm{C}$ NMR (75 MHz, DMSO- $\left.d_{6}\right) \delta 165.7,152.6,148.8,145.3,128.8$, 127.7, 126.7, 99.7, 59.6, 54.4, 18.7,14.5. Anal. C 64.57\%, H $6.12 \%, \mathrm{~N} 10.71 \%$, calcd for $\mathrm{C}_{14} \mathrm{H}_{16} \mathrm{~N}_{2} \mathrm{O}_{3}, \mathrm{C} 64.60 \%$, H $6.20 \%$, N $10.76 \%$.

5-(Ethoxycarbonyl6-methyl)-4-(4-hydroxyphenyl)-3,4-dihydropyrimidin-2(1H)-one (1b). White solid; mp 228-230 ${ }^{\circ} \mathrm{C}$ (Lit. 228-230 ${ }^{\circ} \mathrm{C}$ ) [52]; FT-IR (KBr) $v_{\max } 3513,3362,3278$, $3239,3118,2982,2814,1685,1649,1596,1516,1461,1383$, 1317, 1229, 1171, 1091, 825, 753, 651, 544, $507 \mathrm{~cm}^{-1} ;{ }^{1} \mathrm{H}$ NMR (300 MHz, DMSO- $\left.d_{6}\right) \delta 9.77(2 \mathrm{H}, \mathrm{s}, \mathrm{NH}$ and $\mathrm{OH}), 9.32$ $(1 \mathrm{H}, \mathrm{s}, \mathrm{NH}), 7.09\left(2 \mathrm{H}, \mathrm{d}, J=8.9 \mathrm{~Hz}, \mathrm{C}_{6} \mathrm{H}_{5}\right), 6.74(2 \mathrm{H}, \mathrm{d}, J=8.9$ $\left.\mathrm{Hz}, \mathrm{C}_{6} \mathrm{H}_{5}\right), 5.12(1 \mathrm{H}, \mathrm{s}, \mathrm{CH}), 3.97\left(2 \mathrm{H}, \mathrm{q}, J=6.0 \mathrm{~Hz}, \mathrm{OCH}_{2} \mathrm{CH}_{3}\right)$, $2.27\left(3 \mathrm{H}, \mathrm{s}, \mathrm{CH}_{3}\right), 1.08\left(3 \mathrm{H}, \mathrm{t}, J=6.0 \mathrm{~Hz}, \mathrm{OCH}_{2} \mathrm{CH}_{3}\right)$; Anal. $\mathrm{C}$
$60.79 \%$, H 5.66\%, N $10.09 \%$, calcd for $\mathrm{C}_{14} \mathrm{H}_{16} \mathrm{~N}_{2} \mathrm{O}_{4}, \mathrm{C} 60.86 \%$, H $5.84 \%$, N $10.14 \%$.

5-(Ethoxycarbonyl)-6-methyl-4-(4-methylphenyl)-3,4-dihydropyrimidin-2(1H)-one (1c). White solid; mp 209-211 ${ }^{\circ} \mathrm{C}$ (Lit. 209-211 ${ }^{\circ} \mathrm{C}$ ) [53]; FT-IR (KBr) $v_{\max } 3245$, 3115, 2980 , 2953, 1728, 1704, 1649, 1461, 1422, 1328, 1287, 1222, 1089, 785, $478 \mathrm{~cm}^{-1} ;{ }^{1} \mathrm{H}$ NMR (300 MHz, DMSO- $\left.d_{6}\right) \delta 9.17(1 \mathrm{H}, \mathrm{s}$, $\mathrm{NH}), 7.70(1 \mathrm{H}, \mathrm{s}, \mathrm{NH}), 7.13\left(4 \mathrm{H}, \mathrm{s}, \mathrm{C}_{6} \mathrm{H}_{4}\right), 5.11(1 \mathrm{H}, \mathrm{d}, J=3.0$ $\mathrm{Hz}, \mathrm{CH}), 3.99\left(2 \mathrm{H}, \mathrm{q}, J=6.0 \mathrm{~Hz}, \mathrm{OCH}_{2} \mathrm{CH}_{3}\right), 2.27\left(3 \mathrm{H}, \mathrm{s}, \mathrm{CH}_{3}\right)$, $2.25\left(3 \mathrm{H}, \mathrm{s}, \mathrm{CH}_{3}\right), 1.11\left(3 \mathrm{H}, \mathrm{t}, J=6.0 \mathrm{~Hz}, \mathrm{OCH}_{2} \mathrm{CH}_{3}\right) ;{ }^{13} \mathrm{C}$ NMR $\left(75 \mathrm{MHz}, \mathrm{DMSO}-d_{6}\right) \delta 165.8,152.6,148.6,142.4,136.8$, 129.3, 126.6, 99.8, 59.6, 54.1, 21.1, 18.2, 14.6. Anal. C 65.70\%, $\mathrm{H} 6.58 \%, \mathrm{~N} 10.20 \%$, calcd for $\mathrm{C}_{15} \mathrm{H}_{18} \mathrm{~N}_{2} \mathrm{O}_{3}, \mathrm{C} 65.68 \%, \mathrm{H}$ $6.61 \%$, N $10.21 \%$.

5-(Ethoxycarbonyl)-6-methyl-4-(3-metoxyphenyl)-3,4-dihydropyrimidin-2(1H)-one (1d). White solid; $\mathrm{mp} 200-203{ }^{\circ} \mathrm{C}$ (Lit. $200-202{ }^{\circ} \mathrm{C}$ ) [54]; FT-IR (KBr) $v_{\max } 3449,3268,3245$, 3116, 3014, 2961, 2937, 1704, 1651, 1599, 1493, 1330, 1286, 1227, 1094, 1039, 790, 744, $453 \mathrm{~cm}^{-1}$; ${ }^{1} \mathrm{H}$ NMR $(300 \mathrm{MHz}$, DMSO- $\left.d_{6}\right) \delta 9.20(1 \mathrm{H}, \mathrm{s}, \mathrm{NH}), 7.74(1 \mathrm{H}, \mathrm{s}, \mathrm{NH}), 7.26(1 \mathrm{H}, \mathrm{t}, J=$ $\left.6.0 \mathrm{~Hz}, \mathrm{C}_{6} \mathrm{H}_{4}\right), 6.85-6.79\left(3 \mathrm{H}, \mathrm{m}, \mathrm{C}_{6} \mathrm{H}_{4}\right), 5.14(1 \mathrm{H}, \mathrm{d}, J=3.0$ $\mathrm{Hz}, \mathrm{CH}), 4.01\left(2 \mathrm{H}, \mathrm{q}, J=6.0 \mathrm{~Hz}, \mathrm{OCH}_{2} \mathrm{CH}_{3}\right), 3.73(3 \mathrm{H}, \mathrm{s}$, $\left.\mathrm{OCH}_{3}\right), 2.25\left(3 \mathrm{H}, \mathrm{s}, \mathrm{CH}_{3}\right), 1.12\left(3 \mathrm{H}, \mathrm{t}, J=6.0 \mathrm{~Hz}, \mathrm{OCH}_{2} \mathrm{CH}_{3}\right)$; Anal. C $64.00 \%$, H $6.21 \%$, N 9.47\%, calcd for $\mathrm{C}_{15} \mathrm{H}_{18} \mathrm{~N}_{2} \mathrm{O}_{4}, \mathrm{C}$ $64.06 \%$, H $6.25 \%$, N 9.65\%.

5-(Ethoxycarbonyl)-4-(3-chlorophenyl)-6-methyl-3,4-dihydropyrimidin--2(1H)-one (1e). White solid; mp 190-192 ${ }^{\circ} \mathrm{C}$ (Lit. 190-192 ${ }^{\circ} \mathrm{C}$ ) [52]; FT-IR (KBr) $v_{\max } 3438,3301,3223$, $3120,2980,2928,1707,1662,1612,1588,1528,1475,1376$, 1286, 1227, 1122, 1090, 1022, 870, 783, 691, 605, $446 \mathrm{~cm}^{-1}$; ${ }^{1} \mathrm{H}$ NMR $\left(300 \mathrm{MHz}\right.$, DMSO- $\left.d_{6}\right) \delta 9.29(1 \mathrm{H}, \mathrm{s}, \mathrm{NH}), 7.82(1 \mathrm{H}$, $\mathrm{s}, \mathrm{NH}), 7.41-7.20\left(4 \mathrm{H}, \mathrm{m}, \mathrm{C}_{6} \mathrm{H}_{4}\right), 5.16(1 \mathrm{H}, \mathrm{s}, \mathrm{CH}), 4.01(2 \mathrm{H}, \mathrm{q}$, $\left.J=3.0 \mathrm{~Hz}, \mathrm{OCH}_{2} \mathrm{CH}_{3}\right), 2.27\left(3 \mathrm{H}, \mathrm{s}, \mathrm{CH}_{3}\right), 1.11(3 \mathrm{H}, \mathrm{t}, J=6.0$ $\mathrm{Hz}, \mathrm{OCH}_{2} \mathrm{CH}_{3}$ ); Anal. C $56.97 \%$, H 5.12\%, N 9.39\%, calcd for $\mathrm{C}_{14} \mathrm{H}_{15} \mathrm{ClN}_{2} \mathrm{O}_{3}$, C 57.05\%, H 5.13\%, N 9.50\%.

5-(Ethoxycarbonyl)-4-(3-bromophenyl)-6-methyl-3,4-dihydropyrimidin--2(1H)-one (1f). White solid; mp 196-199 ${ }^{\circ} \mathrm{C}$ (Lit. 196-198 ${ }^{\circ} \mathrm{C}$ ) [55]; FT-IR (KBr) $v_{\max } 3436,3299,3239$, 3120, 2976, 2937, 1705, 1658, 1612, 1589, 1527, 1473, 1377, $1285,1226,1090,782,693,603,456 \mathrm{~cm}^{-1} ;{ }^{1} \mathrm{H}$ NMR $(300$ MHz, DMSO- $\left.d_{6}\right) \delta 9.34(1 \mathrm{H}, \mathrm{s}, \mathrm{NH}), 7.86(1 \mathrm{H}, \mathrm{s}, \mathrm{NH}), 7.35-$ $7.40\left(4 \mathrm{H}, \mathrm{m}_{,} \mathrm{C}_{6} \mathrm{H}_{4}\right), 5.20(1 \mathrm{H}, \mathrm{d}, J=3.0 \mathrm{~Hz}, \mathrm{CH}), 4.07(2 \mathrm{H}, \mathrm{q}$, $\left.J=6.0 \mathrm{~Hz}, \mathrm{OCH}_{2} \mathrm{CH}_{3}\right), 2.32\left(3 \mathrm{H}, \mathrm{s}, \mathrm{CH}_{3}\right), 1.17(3 \mathrm{H}, \mathrm{t}, J=6.0$ $\mathrm{Hz}, \mathrm{OCH}_{2} \mathrm{CH}_{3}$ ); ${ }^{13} \mathrm{C}$ NMR (75 MHz, DMSO- $\left.d_{6}\right) \delta 158.0,149.4$, 146.1, 131.2, 130.7, 130.2, 129.6, 129.1, 99.1, 59.0, 54.0, 18.3,14.5. Anal. C 49.51\%, H 4.32\%, N 8.09\%, calcd for $\mathrm{C}_{14} \mathrm{H}-$ ${ }_{15} \mathrm{BrN}_{2} \mathrm{O}_{3}, \mathrm{C} 49.58 \%, \mathrm{H} 4.46 \%$, N 8.26\%.

5-(Ethoxycarbonyl)-4-(4-fluorophenyl)-6-methyl-3,4-dihydropyrimidin-2(1H)-one (1g). White solid; $\mathrm{mp} 176-178{ }^{\circ} \mathrm{C}$ (Lit. 176-178 ${ }^{\circ} \mathrm{C}$ ) [52]; FT-IR (KBr) $v_{\max } 3445,3337,3247$, 3121, 2980, 2953, 2932, 1727, 1702, 1648, 1600, 1508, 1463, 
$1290,1221,1161,1089,840,793,606,592,508,459 \mathrm{~cm}^{-1} ;{ }^{1} \mathrm{H}$ NMR $\left(300 \mathrm{MHz}, \mathrm{DMSO}-d_{6}\right) \delta 9.28(1 \mathrm{H}, \mathrm{s}, \mathrm{NH}), 7.79(1 \mathrm{H}, \mathrm{s}$, $\mathrm{NH}), 7.28\left(2 \mathrm{H}, \mathrm{t}, J=9.0 \mathrm{~Hz}, \mathrm{C}_{6} \mathrm{H}_{4}\right), 7.15(2 \mathrm{H}, \mathrm{t}, J=9.0 \mathrm{~Hz}$, $\left.\mathrm{C}_{6} \mathrm{H}_{4}\right), 5.18(1 \mathrm{H}, \mathrm{s}, \mathrm{CH}), 3.99\left(2 \mathrm{H}, \mathrm{q}, J=6.0 \mathrm{~Hz}, \mathrm{OCH}_{2} \mathrm{CH}_{3}\right)$, $2.28\left(3 \mathrm{H}, \mathrm{s}, \mathrm{CH}_{3}\right), 1.09\left(3 \mathrm{H}, \mathrm{t}, J=6.0 \mathrm{~Hz}, \mathrm{OCH}_{2} \underline{\mathrm{CH}}_{3}\right)$; Anal. C $60.23 \%, \mathrm{H} 5.12 \%, \mathrm{~N} 10.11 \%$, calcd for $\mathrm{C}_{14} \mathrm{H}_{15} \mathrm{FN}_{2} \mathrm{O}_{3}, \mathrm{C}$ $60.43 \%$, H $5.43 \%$, N $10.07 \%$.

5-(Ethoxycarbonyl)-6-methyl-4-(4-nitrophenyl)-3,4-dihydropyrimidin-2(1H)-one (1h). Pall yellow solid; mp 174-178 ${ }^{\circ} \mathrm{C}$ (Lit. $175-177.5^{\circ} \mathrm{C}$ ) [56a]; FT-IR (KBr) $v_{\max }$ 3485, 3314, 3108, 2978, 2937, 1732, 1711, 1677, 1601, 1568, 1527, 1371, 1351, 1298, 1244, 1177, 1106, 1014, 856, 699, $590 \mathrm{~cm}^{-1} ;{ }^{1} \mathrm{H}$ NMR $\left(300 \mathrm{MHz}, \mathrm{DMSO}-d_{6}\right) \delta 9.41(1 \mathrm{H}, \mathrm{s}, \mathrm{NH}), 8.14(2 \mathrm{H}, \mathrm{d}$, $\left.J=9.0 \mathrm{~Hz}, \mathrm{C}_{6} \mathrm{H}_{4}\right), 7.50(1 \mathrm{H}, \mathrm{s}, \mathrm{NH}), 7.42(2 \mathrm{H}, \mathrm{d}, J=9.0 \mathrm{~Hz}$, $\left.\mathrm{C}_{6} \mathrm{H}_{4}\right), 5.14(1 \mathrm{H}, \mathrm{s}, \mathrm{CH}), 4.09\left(2 \mathrm{H}, \mathrm{q}, J=6.0 \mathrm{~Hz}, \mathrm{OCH}_{2} \mathrm{CH}_{3}\right)$, $2.20\left(3 \mathrm{H}, \mathrm{s}, \mathrm{CH}_{3}\right), 1.19\left(3 \mathrm{H}, \mathrm{t}, J=6.0 \mathrm{~Hz}, \mathrm{OCH}_{2} \mathrm{CH}_{3}\right)$; Anal. C $54.98 \%, \mathrm{H} 4.99 \%$, N $13.63 \%$, calcd for $\mathrm{C}_{14} \mathrm{H}_{15} \mathrm{~N}_{3} \mathrm{O}_{5}, \mathrm{C} 55.08 \%$, H $4.95 \%$, N $13.76 \%$.

5-(Ethoxycarbonyl)-4-(furan-2-yl)-6-methyl-3,4-dihydropyrimidin--2(1H)-one (1i). White solid; mp 194-196 ${ }^{\circ} \mathrm{C}$ (Lit. $196^{\circ} \mathrm{C}$ ) [56b]; FT-IR (KBr) $v_{\max } 3335,3251,3120,2965,2928$, 2851, 1703, 1650, 1457, 1380, 1236, 1098, 798, $465 \mathrm{~cm}^{-1} ;{ }^{1} \mathrm{H}$ NMR (300 MHz, DMSO-d $) \delta 9.17(1 \mathrm{H}, \mathrm{s}, \mathrm{NH}), 7.63(1 \mathrm{H}, \mathrm{d}$, $J=6.0 \mathrm{~Hz}$, furyl), $7.49(1 \mathrm{H}, \mathrm{s}, \mathrm{NH}), 6.28(1 \mathrm{H}, \mathrm{t}, J=3.0 \mathrm{~Hz}$, furyl), $6.02(1 \mathrm{H}, \mathrm{d}, J=3.0 \mathrm{~Hz}$, furyl $), 5.13(1 \mathrm{H}, \mathrm{s}, \mathrm{CH}), 3.96(2 \mathrm{H}$, q, $\left.J=6.0 \mathrm{~Hz}, \mathrm{OCH}_{2} \mathrm{CH}_{3}\right), 2.16\left(3 \mathrm{H}, \mathrm{s}, \mathrm{CH}_{3}\right), 1.07(3 \mathrm{H}, \mathrm{t}, J=6.0$ $\mathrm{Hz}, \mathrm{OCH}_{2} \mathrm{CH}_{3}$ ). Anal. C 57.32\%, H 5.57\%, N $11.21 \%$, calcd for $\mathrm{C}_{12} \mathrm{H}_{14} \mathrm{~N}_{2} \mathrm{O}_{4}, \mathrm{C} 57.59 \%$, H 5.64\%, N $11.19 \%$.

5-(Ethoxycarbonyl)-6-methyl-4-styryl-3,4-dihy-dropyrimidin-2(1H)-one (1g). White solid; mp 238-241 ${ }^{\circ} \mathrm{C}$ (Lit. 240-242 ${ }^{\circ}$ C) [56c]; FT-IR (KBr) $v_{\max }$ 3244, 3112, 3035, 2977, 2932, 1722, 1702, 1652, 1463, 1424, 1387, 1367, 1288, 1227, 1095, $967,779,756,692,488 \mathrm{~cm}^{-1} ;{ }^{1} \mathrm{H}$ NMR (300 MHz, DMSO- $d_{6}$ ) $\delta 9.21(1 \mathrm{H}, \mathrm{s}, \mathrm{NH}), 7.61(1 \mathrm{H}, \mathrm{s}, \mathrm{NH}), 7.28-7.47\left(5 \mathrm{H}, \mathrm{m}, \mathrm{C}_{6} \mathrm{H}_{5}\right)$, $6.43(1 \mathrm{H}, \mathrm{d}, J=15.0 \mathrm{~Hz}, \mathrm{CH}$ vinyl $), 6.25\left(1 \mathrm{H}, \mathrm{dd}, J_{l=18.0} \mathrm{~Hz}\right.$, $J_{2}=6.0 \mathrm{~Hz}, \mathrm{CH}$ vinyl), $4.79\left(1 \mathrm{H}, \mathrm{dd}, J_{l=} 6.0 \mathrm{~Hz}, J_{2}=3.0 \mathrm{~Hz}\right.$, $\mathrm{CH}), 4.08-4.20\left(2 \mathrm{H}, \mathrm{m}, \mathrm{OCH}_{2} \mathrm{CH}_{3}\right), 2.26\left(3 \mathrm{H}, \mathrm{s}, \mathrm{CH}_{3}\right), 1.26$ $\left(3 \mathrm{H}, \mathrm{t}, J=6.0 \mathrm{~Hz}, \mathrm{OCH}_{2} \mathrm{CH}_{3}\right) ;{ }^{2} \mathrm{C} \mathrm{NMR}\left(75 \mathrm{MHz}, \mathrm{DMSO}-d_{6}\right)$ $\delta 165.6,153.0,149.0,136.6,130.4,129.1,128.5,128.0,126.8$, 98.2, 59.6, 52.3, 18.2,14.7. Anal. C 66.97\%, H 6.39\%, N 9.72\%, calcd for $\mathrm{C}_{16} \mathrm{H}_{18} \mathrm{~N}_{2} \mathrm{O}_{3}, \mathrm{C} 67.12 \%$, H 6.34\%, N 9.78\%.

5-Ethoxycarbonyl-6-methyl-4-n-propyl-3,4-dihydropyrimidin-2(1H)-one (1k) White solid; mp 177-180 ${ }^{\circ} \mathrm{C}$ (Lit. 178-180 $\left.{ }^{\circ} \mathrm{C}\right)$ [56d]; FT-IR (KBr) $v_{\max } 3362,3251,3121,2986,2958$, 2937, 1721, 1698, 1674, 1645, 1482, 1464, 1369, 1332, 1286, 1235, 1119, 1090, 1016, 787, 663, $599 \mathrm{~cm}^{-1} ;{ }^{1} \mathrm{H}$ NMR $(300$ $\left.\mathrm{MHz}, \mathrm{DMSO}-d_{6}\right) \delta 8.95(1 \mathrm{H}, \mathrm{s}, \mathrm{NH}), 7.34(1 \mathrm{H}, \mathrm{s}, \mathrm{NH}), 4.12-$ $4.03\left(3 \mathrm{H}, \mathrm{m}, \mathrm{OCH}_{2} \mathrm{CH}_{3}\right.$ and $\left.\mathrm{CH}\right), 2.17\left(3 \mathrm{H}, \mathrm{s}, \mathrm{CH}_{3}\right), 1.45-1.24$ $\left(4 \mathrm{H}, \mathrm{m}, \mathrm{CH}_{2}-\mathrm{CH}_{2}\right), 1.20\left(3 \mathrm{H}, \mathrm{t}, J=6.0 \mathrm{~Hz}, \mathrm{CH}_{3}\right), 0.86(3 \mathrm{H}, \mathrm{t}$, $\left.J=6.0 \mathrm{~Hz}, \mathrm{OCH}_{2} \underline{\mathrm{CH}}_{3}\right) ;{ }^{13} \mathrm{C} \mathrm{NMR}\left(75 \mathrm{MHz}, \mathrm{DMSO}-d_{6}\right) \delta 165.9$, 153.2, 148.7, 99.8, 59.5, 50.2, 18.1, 17.4, 14.6,14.2. Anal. C
$58.32 \%, \mathrm{H} 8.10 \%$, N $12.25 \%$, calcd for $\mathrm{C}_{11} \mathrm{H}_{18} \mathrm{~N}_{2} \mathrm{O}_{3}, \mathrm{C} 58.39 \%$, H $8.02 \%$, N $12.38 \%$.

5-Ethoxycarbonyl-4,6-dimethyl-3,4-dihydropyrimidin-2 (1H)-one (11). White solid; mp 175-178 ${ }^{\circ} \mathrm{C}$ (Lit. $178{ }^{\circ} \mathrm{C}$ ) [56e]; FT-IR (KBr) $v_{\max } 3438,3345,3260,2929,2804,2639,1682$, $1625,1601,1465,1153,1053,1002,788,718,559 \mathrm{~cm}^{-1} ;{ }^{1} \mathrm{H}$ NMR (300 MHz, DMSO-d $)_{6} \delta 9.12(1 \mathrm{H}, \mathrm{s}, \mathrm{NH}), 7.29(1 \mathrm{H}, \mathrm{s}$, $\mathrm{NH})$, 4.19-4.05 (3H, m, $\underline{\mathrm{CH}}_{2} \mathrm{CH}_{3}$ and $\left.\mathrm{CH}\right), 2.19\left(3 \mathrm{H}, \mathrm{s}, \mathrm{CH}_{3}\right)$, 1.25-1.17(6H, m, $\mathrm{OCH}_{2} \mathrm{CH}_{3}$ and $\left.\mathrm{CH}_{3} \mathrm{CH}\right)$; Anal. C 54.19\%, H $7.17 \%, \mathrm{~N} 14.05 \%$, calcd for $\mathrm{C}_{9} \mathrm{H}_{14} \mathrm{~N}_{2} \mathrm{O}_{3}, \mathrm{C} 54.53 \%, \mathrm{H} 7.12 \%$, N $14.13 \%$.

\section{Acknowledgments}

The authors gratefully acknowledge the partial support of this study by the Islamic Azad University of Bojnourd.

\section{References}

1. Kappe, C. O. Tetrahedron 1993, 49, 6937.

2. Kappe, C. O. Acc. Chem. Res. 2000, 33, 879.

3. Kappe, C. O. Eur. J. Med. Chem. 2000, 35, 1043.

4. Atwal, K. S.; Swanson, B. N.; Unger, S. E.; Floyed, D. M.; Moreland, S.; Hedberg, A.; O'Reilly, A. J. Med. Chem. 1991, 34, 806.

5. Adib, M.; Sheikhi, E.; Kavoosi, A.; Bijanzadeh, H.R. Tetrahedron 2010, 66, 9263.

6. Pourjavadi, A.; Salimi, H.; Barzegar, Sh.; Eftekhari, B. Acta Chim. Slov. 2007, 54, 140.

7. Atwal, K. S.; Rovnyak, G. C.; O’Reilly, B. C. Schwartz, J. J. Org. Chem. 1989, 54, 5898 .

8. Tao, L.; Zhu, C.; Wei, Y.; Zhao, Y. Adv. Polym. Sci. 2015, 269, 43.

9. Elnagdi, M. H; Moustafa, M. S.; Al-Mousawi, S. M.; Mekheimer, R. A.; Sadek, K. U. Mol. Divers. 2015, 19, 625.

10. Singh, M. S.; Chowdhury, S. RSC Adv. 2012, 2, 4547.

11. Zhu, J.; Bienayme, H. Eds.; Multicomponent Reactions, Wiley-VCH: Weinheim, Germany, 2005.

12. Dabiri, M.; Noroozi Tisseh, Z.; Bahramnejad, M.; Bazgir, A. Ultrason. Sonochem. 2011, 18, 1153.

13. Hasaninejad, A.; Zare, A.; Shekouhy, M. Tetrahedron 2011, 67, 390.

14. Mirjalili, B. F.; Bamoniri, A.; Akbari, A.; Taghavinia, N. J. Iran. Chem. Soc. 2011, 8, S129.

15. Biginelli, R. Gazz. Chim. Ital. 1893, 23, 360.

16. Nagarajaiah, H.; Mukhopadhyay, A.; Moorthy, J. N. Tetrahedron Lett. 2016, 57, 5135.

17. Alvim, H. G. O. ; da Silva Junior, E. N. ; Neto, B. A. D. RSC Adv. 2014, 4, 54282.

18. Sandhu, S. ; Sandhu, J. S. Arkivoc 2012:, 66.

19. Banik, B. K.; Reddy, A. T.; Datta, A.; Mukhopadhyay, C. Tetrahedron Lett. 2007, 48, 7392.

20. Li, J. T.; Han, J. F.; Yang, J. H.; Li, T. S. Ultrason. Sonochem. 2003, 10, 119.

21. Peng, J. J.; Deng, Y. Q. Tetrahedron Lett. 2001, 42, 5917.

22. Ma, Y.; Qian, C.; Wang, L.; Yang, M. J. Org. Chem. 2000, 65, 3864. 
23. Tu, S. J.; Fang, F.; Miao, C. B.; Jiang, H.; Feng, Y. J.; Shi, D. Q.; Wang, X. S. Tetrahedron Lett. 2003, 44, 6153.

24. Sabitha, G.; Reddy, G. S. K. K.; Reddy, K. B.; Yadav, J. S. Tetrahedron Lett. 2003, 44, 6497.

25. Su, W. K.; Li, J. J.; Zheng, Z. G.; Shen, Y. C. Tetrahedron Lett. 2005, 46, 6037.

26. Hatamjafari, F. Org. Chem. Int. 2014, 4, 1.

27. Fu, N. Y.; Yuan, Y. F.; Pang, M. L.; Wang, J. T.; Peppe, C. J. Organomet. Chem. 2003, 672, 52.

28. Azizian, J.; Mohammadi, A. A.; Karimi, A. R.; Mohammadizadeh, M. R. Appl. Catal., A Gen. 2006, 300, 85.

29. Salehi, P.; Dabiri, M.; Zolfigol, M. A.; Fard, M. A. B. Tetrahedron Lett. 2003, 44, 2889.

30. Kumar, K. A.; Kasthuraiah, M.; Reddy, C. S.; Reddy, C. D. Tetrahedron Lett. 2001, 42, 7873.

31. Nandurkar, N. S.; Bhanushali, M. J.; Bhor, M. D.; Bhanage, B. M. J. Mol. Catal. A: Chem. 2007, 271, 14.

32. Tajbakhsh, M.; Mohajerani, B.; Heravi, M. M.; Ahmadi, A. N. J. Mol. Catal. A: Chem. 2005, 236, 216.

33. Ahmed, N.; van Lier, J. E. Tetrahedron Lett. 2007, 48, 5407.

34. Adib, M.; Ghanbary, K.; Mostofi, M.; Ganjali, M. R. Molecules 2006, 11, 649 .

35. Abbasi, E.; Hatamjafari, F. Orient. J. Chem. 2013, 29, 731.

36. Gharib, A.; Jahangir, M.; Roshani, M.; Scheeren, J. W.; Moghadaszadeh, S.; Rezaee, A. GU J Sci. 2012, 25, 823.

37. Patil, S.; Jadhav, S. D.; Deshmukh, M. B. Arch. Appl. Sci. Res. 2011, 3, 203.

38. Sheldon, R. A.; van Bekkum H. Eds.; Fine Chemical through Heterogeneous Catalysis. Wiley-VCH: Weinheim, Germany, 2002.

39. Cadman, J.; Zhou, S.; Chen, Y.; Li, Q. J. Bionic Eng. 2012, 9, 367.

40. Birchall, J. D.; Thomas, N. L. J. Mater. Sci. 1983, 18, 2081.

41. Gower, D.; Vincent, J. F. V. Biomimetics, 1996, 4, 37.
42. Sherrard, K. M. Biol. Bull. 2000, 198, 404.

43. Falini, G.; Fermani, S. Tissue Eng. 2004, 10, 1.

44. Ward, P. D.; Boletzky, S. V. J. Mar. Biol. Assoc. U. K. 1984, 64, 955.

45. Vogel, S. J. Biosciences. 2006, 31, 309.

46. Cadman, J.; Chen, Y.; Zhou, Sh.; Li, Q. Adv. Mater. Res. 2010, 123,295

47. Jothi, N.; Kunthavai Nachiyar, R. Global J. Biotechnol. Biochem. 2013, 8, 33 .

48. Ghodsinia, S. E.; Akhlaghinia, B. RSC Adv. 2015, 5, 49849.

49. Osada, M.; Miura, C.; Nakagawa, Y. S.; Kaihara, M.; Nikaido, M.; Totani, K. Carbohydr. Polym. 2013, 92, 1573.

50. Pearson, F. G.; Marchessault, R. H.; Liang, C. Y. J. Polym. Sci. 1960, 43, 101.

51. Khodja, I. A.; Boulcina, R.; Debache, A. Lett. Org. Chem. 2015, 12,77 .

52. Mohamadpour, F.; Maghsoodlou, M. T.; Heydari, R.; Lashkari, M. J. Iran. Chem. Soc. 2016, 13, 1549.

53. Liu, Z.; Ma, R.; Cao, D.; Liu, C. Molecules 2016, 21, 462.

54. Fazaeli, R.; Aliyan, H.; Mohammadifar, F.; Zamani, A. A.; Bagi, M.J. J. Korean. Chem. Soc. 2011, 55, 666.

55. Dadhania, A. N.; Patel, V. K.; Raval, D. K. J. Chem. Sci, 2012, $124,921$.

56. (a) Foroughifar, N.; Mobinikhaledi, A.; Fathinejad, H. Phosphorus, Sulfur Silicon Relat. Elem. 2003, 178, 495. (b) Firdous, H.; Zafar, A. M.; Khan, M. N.; Parveen, N.; Munawar, A. M.; Khan, M. A. Asian J. Chem. 2015, 27, 455. (c) Salehi, H.; Guo, Q.X. Synth. Commun. 2004, 34, 171. (d) Nasr-Esfahani, M.; Montazerozohori, M.; Aghel-Mirrezaee, M.; Kashi, H. J. Chil. Chem. Soc. 2014, 59, 2311. (e) Ramos, L. M.; Guido, B. C.; Nobrega, C. C.; Correa, J. R.; Silva, R. G.; De Oliveira, H. C. B.; Gomes, A. F.; Gozzo, F. C.; Neto, B. A. D. Chem. Eur. J. 2013, 19, 4156. 